\title{
Acute Myocarditis in Childhood and Adolescence in the Covid-19 Era
}

\author{
Ana Flávia Malheiros Torbey, ${ }^{1}$ [0 Aurea Lúcia Alves de Azevedo Grippa de Souza, ${ }^{10}$ Ana Catarina Durán Bustamante, ${ }^{10}$ \\ Carmen Z. Brandão, ${ }^{10}$ Luan Rodrigues Abdallah, ${ }^{10}$ Yves P. D. March e Souza, $^{1 \text { (이 }}$ Evandro Tinoco Mesquita $^{10}$ \\ Universidade Federal Fluminense, ${ }^{1}$ Niterói, RJ - Brazil
}

\begin{abstract}
Myocarditis in children and adolescents is an inflammatory myocardial disease with a heterogeneous presentation ranging from oligosymptomatic, with no impaired ejection fraction to catastrophic clinical presentation with cardiogenic shock (fulminant myocarditis) or sudden death. Thus, this condition remains a major challenge from the diagnostic and therapeutic points of view. Despite the different etiologies listed, the most frequent form may be related to viral diseases, and new forms of myocarditis, such allergic ones (eosinophilic and use of medications, particularly cancer immunotherapy), have been currently described. Increasing the rate of suspicion is crucial. Measurement of serum levels of troponin and $\mathrm{N}$-terminal B-type natriuretic peptide, as well as the use of advanced methods of cardiac imaging, such as strain echocardiogram, positron emission tomography, and cardiac magnetic resonance, are integral part of multimodal assessment in suspected patients. Although endomyocardial biopsy remains is still the gold standard for disease diagnosis, currently it tends to be performed in specific situations, such as fulminant clinical presentation and chronic or recurrent myocarditis. The management of this condition includes general supportive care, treatment of heart failure, control of arrhythmias when present, assessment of mechanical support and cardiac transplantation. Treatments based on immunosuppression and immunotherapy are still controversial. In this article, we propose a broad review of diagnostic and treatment methods, as well as the different etiologies in the pediatric population.
\end{abstract}

\section{Introduction}

Myocarditis represents a heterogeneous group of myocardial inflammatory diseases in terms of etiology, inflammatory response, clinical course, and response to treatment. Additionally, it shows high morbidity and mortality rates in the pediatric population ${ }^{1}$ and is considered the most common cause of heart failure (HF) in previously healthy children. ${ }^{1,2}$ Furthermore, its clinical presentation has a spectrum of signs and symptoms ranging from oligosymptomatic cases to cardiogenic shock and sudden death, which represents a

\section{Keywords}

Myocarditis; Cardiomyopathy; Heart Failure; Children; Adolescents.

Correspondência: Ana Flávia Malheiros Torbey •

Hospital Universitário Antônio Pedro, Faculdade de Medicina,

Departamento Materno Infantil - Rua Marquês de Paraná, 303.

Postal Code 24033-900, Centro, Niterói, RJ - Brazil

E-mail: anatorbey@id.uff.br

Manuscript received April 28, 2021, revised manuscript May 26,2021, accepted June 20,2021 diagnostic challenge in pediatrics. ${ }^{3}$ Dilated cardiomyopathy (DCM), a severe complication of myocarditis, is a common cause of heart transplantation in childhood. ${ }^{1,2,4}$

This article aims to present the advances in the field of diagnosis and treatment, in order to propose an algorithm to aid pediatricians, family physicians, and pediatric cardiologists in approaching patients with myocarditis.

\section{Definition}

Myocarditis is characterized by myocardial inflammation associated with a process of myocyte degeneration and necrosis, may be focal or diffuse, and may cause cardiac dysfunction and/or arrhythmias. ${ }^{1}$ In 1995, myocarditis was classified as an inflammatory cardiomyopathy by the World Health Organization, and its diagnosis is established based on histopathological, immunological and immunohistochemical criteria. Moreover, infectious causes, toxic agents, autoimmune and autoinflammatory conditions, allergic reactions, or idiopathic causes may be involved, ${ }^{5}$ thus characterizing the heterogeneous nature of the disease.

\section{Epidemiology}

The incidence and prevalence of myocarditis are difficult to determine, due to its varied clinical presentation. ${ }^{1,6,7}$ A population-based registry study conducted by Arola et al. ${ }^{8}$ in Finland observed an incidence of myocarditis of 1.95:100 000 people/year (considering children and adolescents from 0 to 15 year old).

Despite its rarity, myocarditis is significant, ${ }^{3}$ since most patients require admission at intensive care unit, as well and ventilatory and circulatory supports. ${ }^{6}$ This condition is responsible for 0.5 cases for each 10000 consultations in pediatric emergency; ${ }^{1}$ however, the actual frequency is probably higher than that reported, due to undiagnosed or asymptomatic cases. ${ }^{8}$

In international studies not including Latin American countries, the age distribution of disease diagnosis is bimodal, with a peak in the first year of life and another at 16 years of age. In adolescents, the disease is more frequent in the male sex $(81 \%)$ than in the female..$^{8,9}$ Also according to the work performed by Arola et al., ${ }^{8}$ the incidence of myocarditis among male adolescents may be as high as 18.1:100 000 people/ year. This increased incidence among male adolescents may be justified by the presence of testosterone.

Although outcome is favorable in nearly $50 \%$ of patients, morbidity and mortality rates remain significant. Sequelae and chronic evolution occur in approximately $30 \%$ of the cases, with $80 \%$ of cases of chronic cardiomyopathy leading to cardiac transplantation or death. ${ }^{5}$ An autopsy study with

DOI: https://doi.org/10.36660/abchf.20210008 
pediatric patients observed that $2 \%$ of deaths were caused by myocarditis. ${ }^{10}$ In a recent autopsy pediatric study, $1.8 \%$ of patients had histological evidence compatible with myocarditis; of these, $57 \%$ had sudden death and 54\% were younger than 1 year of age. ${ }^{11}$

Myocarditis accounts for $30 \%$ to $35 \%$ of children with a phenotype of DCM in registers from Australia and North America and for $22 \%$ of new-onset left ventricular dysfunction in the United Kingdom. ${ }^{12-14}$

Myocarditis in children is associated with higher rates of $\mathrm{HF}$, hospitalization, length of stay at intensive care unit, and use of inotropic support at the time of diagnosis compared to children with idiopathic DCM. A recent study of hospitalized patients in the United States showed that almost a half of patients required inotropic support, $37.5 \%$ required mechanical ventilation, and $7.4 \%$ required extracorporeal membrane oxygenation (ECMO) support. ${ }^{15,16}$

Mortality at the acute phase ranges from 7 to $15 \%$ of the cases, and myocarditis is an important cause of DCM and cardiac transplantation in children without congenital heart disease, which account for $80 \%$ of the cases. ${ }^{6}$

\section{Pathophysiology}

Although the pathogenesis of myocarditis will depend on its etiology, much of our understanding on the disease is based on experimental models of guinea pigs infected with coxsackievirus. ${ }^{17}$ In viral myocarditis, the existence of three phases has been proposed. Initially, there is a period of viral replication, leading to acute myocyte injury and consequently evolving to DCM, which is associated with changes in the extracellular matrix of the myocardial tissue. ${ }^{1}$

\section{Etiology}

A wide spectrum of infectious and immunomediated causes, as well as hypersensitivity to medications, drugs, and toxins, are involved in the etiology of myocarditis. ${ }^{1,18-20}$ Most cases in the pediatric population are caused by viral infections, mainly with adenovirus, cytomegalovirus, EpsteinBarr virus, and enterovirus, the main of which being coxsackie virus type B. However, with the emergence of new diagnostic modalities, other pathogens were also detected, including polymerase chain reaction (PCR) and endomyocardial biopsy (EMB), parvovirus B19, human herpes virus 6, in several patients with myocarditis confirmed by biopsy in European studies. ${ }^{18}$ Arboviruses, such as Chikungunya, dengue, and Zika virus, may also be involved. ${ }^{21}$ Recently, cases of myocarditis due to H1N1 infection were documented. ${ }^{1}$

Thus, infectious exposures, especially to viruses, are responsible for most cases of pediatric myocarditis in Canada and in the United States; ${ }^{19}$ whereas the most common cause of this condition worldwide is Trypanosoma cruzi. It is worth emphasizing that, in Latin America, chagasic myocarditis may affect children and adolescents in endemic areas and, due to migration, patients from these areas may be diagnosed in North America, Europe, and Asia. ${ }^{5,19}$

Currently, in view of the pandemic caused by the coronavirus disease 2019 (COVID-1), cases of myocarditis related to the severe acute respiratory syndrome virus 2 (SARS-CoV-2) have been described. ${ }^{22,23}$ In pediatrics, myocarditis has shown to be related to cases of multi-systemic inflammatory syndrome and associated with clinical signs of Kawasaki disease. ${ }^{22}$ In this cases, troponin elevation is one of main markers of myocardial involvement. ${ }^{23,24}$ Dolhnikof et al. ${ }^{25}$ showed the presence of particles of SARS-CoV-2 in the myocardial tissue through an electronic microscopic study and detection of viral RNA with reverse transcriptase PCR (RT-PCR). Multisystem inflammatory syndrome in children occurs some weeks after SARS-CoV-2 infection; therefore, patients may present with signs of acute HF and negative RT-PCR for SARS-CoV-2, associated with a significant elevation of troponin levels and inflammatory markers. ${ }^{26}$

Autoimmune myocarditis may be an isolated condition, such as in giant cell eosinophilic myocarditis and, or be part of a systemic disease, such as systemic lupus eritematosus, Sjögren syndrome, vasculitis, and polymyositis. ${ }^{27}$ In scenarios of autoimmune myocarditis, there is lymphocytic infiltrate, suggesting important participation of T cells. ${ }^{27}$ Simpson et al. ${ }^{28}$ showed evidence of autoimmune mechanism in children with clinical picture of myocarditis through the presence of significant elevation of human cardiac anti-myosin antibodies. ${ }^{28}$

In addition to chemotherapy and radiation therapy used in the treatment of childhood cancer, new therapies with immunotherapy - checkpoint inhibitors - characterize potential risks for cardiac toxicity and are related to cases of myocarditis. ${ }^{29-31}$ These patients should receive special attention, because they may initially present with normal echocardiogram, and more complex diagnostic methods are needed to confirm myocardial inflammation. ${ }^{31}$ The Table 1 shows the different causes of myocarditis.

\section{Clinical presentation}

The diagnosis of pediatric myocarditis remains a challenge, due to its varied clinical presentation, which ranges from asymptomatic patients with only subtle findings on electrocardiogram (ECG) to fulminant HF and sudden death (MAY 2011). Thus, initial clinical suspicion is necessary for the diagnosis to be confirmed, especially in oligosymptomatic cases.

Studies such as those by Rodrigues-Gonzales et al. ${ }^{6}$ and Durani et al. ${ }^{4}$ showed that, in most patients (59 to $83 \%$ ), the diagnosis of myocarditis was not made on the initial assessment; thus, more than one medical assessment were required before the diagnosis of myocarditis or DCM was made. ${ }^{18}$ In the studies, children often received an initial diagnosis of respiratory disease (bronchiolitis, asthma, or pneumonia) or gastrointestinal infection. ${ }^{1}$

Clinical presentation also varies according to patient's age. Neonates and infants may present with unspecific symptoms suggestive of infection, including fever, hypoactivity, and suction difficulty, or may severe signs, such apneia, cyanosis, and cardiogenic shock. ${ }^{2,7}$

In older children and in adolescents, clinical manifestations may also be different. Most children present with unspecific respiratory or gastrointestinal complaints; only a minority of 


\begin{tabular}{|c|c|c|}
\hline Etiology & & Examples \\
\hline \multirow{4}{*}{ Infectious } & Viruses & $\begin{array}{l}\text { Adenovirus, echovirus, enterovirus (coxsackie), herpes (cytomegalovirus, Epstein-Barr virus, } \\
\text { human herpes virus type } 6 \text {, hepatitis C, HIV, H1N1, parvovirus B19, arboviruses (dengue, } \\
\text { Chikungunya, and Zika), SARS-CoV-2 }\end{array}$ \\
\hline & Bacteria & $\begin{array}{l}\text { Clamidia, Corynebacterium diphtheriae, Klebsiella, Salmonella, Legionella, Mycobacterium } \\
\text { tuberculosis, micoplasma, Staphylococcus, Streptococcus A, Streptococcus pneumonia, } \\
\text { Tryponema pallidum, Haemophilus influenzae }\end{array}$ \\
\hline & Fungi & Actinomyces, Aspergillus, Candida, Cryptococcus \\
\hline & Protozoa & Toxoplasma gondii, Trypanosoma cruzi \\
\hline Autoimmune & \multicolumn{2}{|c|}{$\begin{array}{l}\text { Celiac disease, Churg-Strauss syndrome, dermatomyositis, giant cell myocarditis, hypereosinophilic syndrome, } \\
\text { Kawasaki disease, systemic lupus eritematosus, lymphofollicular myocarditis, rheumatoid arthritis, sarcoidosis, } \\
\text { ulcerative colitis, scleroderma }\end{array}$} \\
\hline \multirow[t]{2}{*}{ Hypersensitivity reactions } & \multicolumn{2}{|c|}{$\begin{array}{l}\text { Penicillin, ampicillin, cephaloesporin, tetracyclines, sulfonamides, benzodiazepines, clozapine, thiazide and loop } \\
\text { diuretics, methyldopa, tetanus toxin, varicella vaccine, antidepressants }\end{array}$} \\
\hline & \multicolumn{2}{|c|}{$\begin{array}{l}\text { Anphetamines, anthracyclines, catecholamine, cocaine, cyclophosphamide, phenytoin, transtuzumab, toxic } \\
\text { amitriptyline, amphotericin B, cannabis, carbon monoxide, electric shock, ethanol, Hymenoptera, isoniazid, } \\
\text { lidocaine, methyldopa, spider and scorpion venoms }\end{array}$} \\
\hline
\end{tabular}

HIV: human immunodeficiency virus; SARS-CoV-2: severe acute respiratory syndrome coronavirus 2.

them report chest pain. The most frequently reported signs of myocarditis are unspecific symptoms, such as shortness of breath (69\%), vomiting (48\%), or feeding difficulties (40\%). Sudden death may occur in some cases of myocarditis. ${ }^{2}$ Every patient with new-onset HF or arrhythmia should have myocarditis considered as a possible diagnosis.

The presentation of myocarditis may be categorized into clinical syndromes such as: chest pain, HF with phenotype of DCM or fulminant myocarditis, arrhythmias, and sudden death.

Chest pain: intense chest pain, similar to that of acute myocardial infarction, associated with typical electrocardiographic changes and increase in biomarkers such as troponin and brain natriuretic peptide (BNP). ${ }^{1}$ It is usually associated with pericardial impairment, but cardiac function is normal. ${ }^{32}$ In these patients, it is necessary to ensure that coronaries do not have congenital or acquired anatomical abnormalities through imaging tests such as echocardiogram and coronary computed tomography angiography.

Sudden death: despite being a rare event, sudden death in the pediatric population is commonly associated with myocarditis ${ }^{16}$ and may be the initial presentation of the disease. In these cases, diagnosis is made histologically, in which an infiltrative inflammatory process becomes an arrhythmogenic focus generating fatal arrhythmia, or through cardiac imaging after an event of aborted sudden death. Myocarditis accounts for approximately $9 \%$ of sudden deaths in young athletes in the United States with confirmed cardiovascular event was documented. ${ }^{28}$

\section{Arrhythmias}

Symptoms such as palpitations and syncope occur in pediatric patients with myocarditis even in the absence of HF or of overt reduction of left ventricular a function.
Resting sinus tachycardia is also an important subtle characteristic and, despite not being a consistent finding, it may be the only finding in mild forms.

The presence of ventricular arrhythmias in structurally normal hearts and ventricular tachyarrhythmias in athletes has been associated with myocarditis. ${ }^{30,31}$ Myocarditis should also be considered in children with acquired complete heart block. Lyme carditits and Chagas disease have been associated with complete heart block. Although most children may recover atrioventricular conduction, some patients may require permanent. Although most children may recover atrioventricular conduction, some patients may require permanent pacemaker implantation. ${ }^{32-34}$

\section{HF with phenotype of DCM}

The most classical presentation of myocarditis is the onset of symptoms of HF in a phenotype of DCM some weeks after a history compatible with viral disease. Respiratory and gastrointestinal symptoms, as well as myalgia, are characteristic of this phase, and fever may be present or not. ${ }^{19,35}$

Signs of congestive HF are observed in most patients ${ }^{18}$ varying in severity of symptoms according to the degree of myocardial inflammation. ${ }^{1}$ Tachydyspnea, tachycardia, changes in cardiac and respiratory auscultation, and hepatomegaly are usually observed, followed by signs of low cardiac output.

\section{Fulminant acute myocarditis}

Is a different subset of acute myocarditis characterized by HF with severe hemodynamic involvement requiring mechanical or inotropic circulatory support and meeting at least two of the following criteria: fever, onset with symptoms different from those of HF within 1 or 2 days, and history of viral disease in the 2 weeks before hospitalization. Fulminant myocarditis is associated with symptoms of major left ventricular dysfunction and unexpected HF from 2 to 3 weeks after the onset of viral infection. ${ }^{14,36}$ Fulminant myocarditis accounts for 10 to $38 \%$ of all 
cases of myocarditis, ${ }^{37}$ and has been described in children with mortality rates ranging from $48.4 \%$ in Japan to $9 \%$ in France. Despite the severe presentation, results are significantly better that those of adults with acute myocarditis. In children presenting with cardiogenic shock, ventricular arrhythmia, or atrioventricular block stabilization with mechanical circulatory support (MCS) will be required, such as extracorporeal membrane oxygenation (ECMO). In patients who do not respond after a period of 1 to 2 weeks, an endomyocardial biopsy (EMB) should be considered in order to rule out giant cell myocarditis that responds to treatment with immunosuppression, despite having a poor prognosis. ${ }^{38}$

\section{Diagnosis}

The diagnosis of myocarditis is especially challenging due to its varied clinical presentation, which encompasses from asymptomatic patients with only subtle findings on ECG to fulminant HF and sudden death. Diagnosis remains mostly based on high degree of clinical suspicion, supported by biomarkers, such as cardiac troponin and BNP, and advanced techniques of cardiac imaging, such as tissue Doppler echocardiography and cardiac magnetic resonance, with EMB being considered the gold standard of diagnosis. Clinical characteristics of myocarditis are usually similar to that of HF, and this condition is the most common cause of new-onset HF in healthy children. Patients with mild symptoms are at risk of worsening and, thus, early diagnosis is important in the establishment of appropriate monitoring and support care..$^{5,39}$

After clinical suspicion, based on medical history taking and physical examination, investigation begins with supplementary tests. Table 2 and Figure 1 summarize the main diagnostic tools and possible findings.

\section{ECG}

In children with myocarditis, ECG is altered in at least $93 \%$ of patients, ${ }^{20}$ although normal results do not exclude the diagnosis of this condition. Changes are unspecific and range from sinus tachycardia to ventricular arrhythmias, and there may be low QRS complex voltage, atrioventricular block, changes in ST segment and QT interval. ${ }^{15,19,20}$

\section{Biomarkers}

The levels of inflammatory activity markers, such as hemosedimentation velocity and reactive $\mathrm{C}$ protein, are often elevated, and leukocytosis may be present, although normal results do not rule out the diagnosis of myocarditis. ${ }^{11,19}$

Abnormal troponin levels may support the diagnosis of myocarditis. Troponin T may have greater sensitivity to detect micronecrosis in myocarditis, due to the proportionally higher and longer increase in its serum levels compared with creatine kinase. ${ }^{11,35}$ It is believed that EMB should be performed when troponin levels are elevated, because there is a greater likelihood of showing myocardial inflammatory infiltrate. ${ }^{15}$

Soongswang et al. ${ }^{40}$ found that troponin $\mathrm{T}$ has good sensitivity (71\%) and specificity (86\%) to diagnose acute myocarditis in children and that troponin T levels were higher in patients with myocarditis compared to those with DCM. ${ }^{40}$ However, it is important to highlight that, in clinical practice, values of troponin $\mathrm{T}$ and I are measured by different methods; therefore, it is essential to know the cutoff values for the test kits used in the institution where the patient was treated.

High levels of natriuretic peptide (BNP and N-terminal B-type natriuretic peptide [NT-pro-BNP]) may be present in myocarditis and are useful to determine cardiac etiology in children with respiratory symptoms. ${ }^{19}$ Rodriguez-Gonzalez et al. ${ }^{6}$ showed that NT-proBNP levels $>5,000 \mathrm{pg} / \mathrm{mL}$ are associated with worse prognosis in children with myocarditis.

\section{Chest X-RAY}

Findings on chest x-ray usually include increased cardiac area on account of increased left ventricular (LV) diameter and/or pericardial effusion ${ }^{7,20}$ and may reveal pulmonary congestion and edema, infiltrates, or pleural effusion. ${ }^{7}$

Table 2 - Main diagnostic tools in acute myocarditis in children and adolescents

\begin{tabular}{|c|c|}
\hline Investigation tools & Possible findings \\
\hline Medical history taking & $\begin{array}{l}\text { Typical symptoms of cold/influenza, gastroenteritis, insect bites, family history of cardiomyopathy, } \\
\text { and precordial pain. Signs of new-onset heart failure or arrhythmia in previously healthy children. }\end{array}$ \\
\hline Physical examination & Resting tachycardia and clinical signs of heart failure. \\
\hline Biomarkers & Troponin and BNP elevation. \\
\hline Chest x-ray & Image suggestive of increased cardiac area, pulmonary congestion, pleural effusion, and pulmonary infiltrate. \\
\hline Electrocardiogram & $\begin{array}{l}\text { Sinus tachycardia, ventricular hypertrophy, abnormalities in ST segment and in T wave, arrhythmias, AVB, } \\
\text { QT segment prolongation, low QRS complex voltage. }\end{array}$ \\
\hline Echocardiogram & $\begin{array}{l}\text { Increased telesystolic and diastolic ventricular volume, wall motion abnormalities, septal or ventricular } \\
\text { thickening, reduced ejection fraction, valvular regurgitation, and presence of thrombi. }\end{array}$ \\
\hline Gallium scintigraphy & Capture of gallium \\
\hline Nuclear magnetic resonance & Edema, cardiac tissue inflammation and necrosis/fibrosis. \\
\hline Myocardial biopsy & Presence of lymphocytic infiltrate, edema, and necrosis of cardiac muscle cells, viral PCR. \\
\hline
\end{tabular}

AVB: atrioventricular block; BNP: brain natriuretic peptide. 


\section{Review Article}

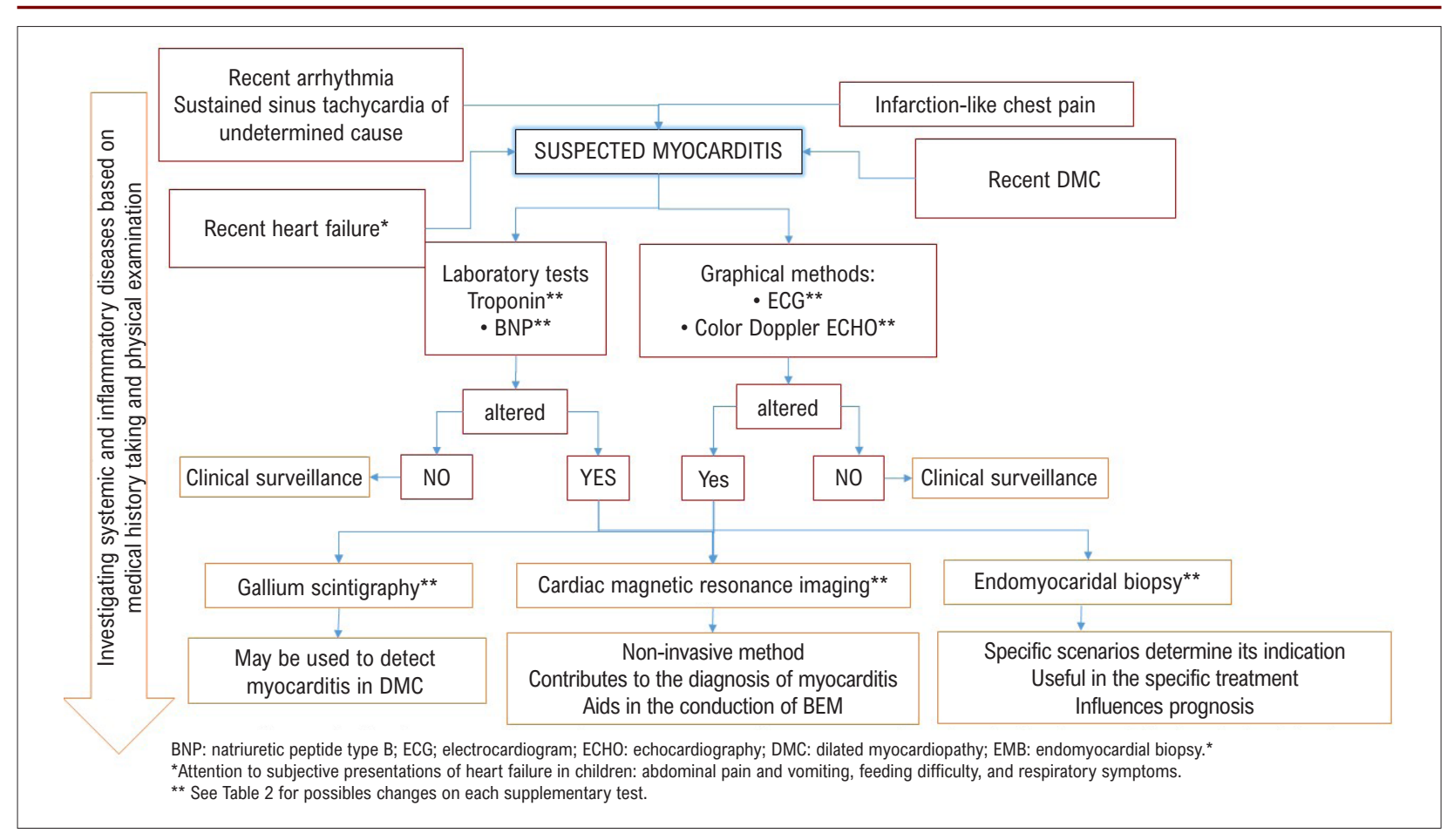

Figure 1 - Diagnostic flowchart for pediatric patients with suspected myocarditis.

\section{Echocardiography}

Echocardiographic findings typical of myocarditis are increased LV systolic and diastolic diameters and reduced LV shortening and ejection fractions, characterizing the phenotype of DCM. ${ }^{19,41}$ Atrioventricular valvular regurgitation and segmental abnormalities of ventricular wall motion, as well as pericardial effusion, may also be observed.,19,41 Changes in right ventricular function may be associated with greater likelihood of adverse outcomes, including cardiac transplantation or death. ${ }^{7}$

Fulminant myocarditis may be characterized by increased intraventricular septal thickness due to interstitial edema and LV dimensions close to normal. ${ }^{19,41}$ Patients with this condition have better prognosis in recovering LV function. ${ }^{19}$ Phenotypes of hypertrophic and restrictive cardiomyopathy have already been described in patients with diagnosis of myocarditis confirmed by histopathology. ${ }^{7,19}$

The use of tissue Doppler and ventricular strain may also contribute in the diagnosis of myocarditis. ${ }^{32,41}$ However, the diagnostic value of $\mathrm{ECHO}$ is limited, because many patients with mild myocarditis may present with normal echocardiographic results. ${ }^{42}$ Echocardiography is also important to rule out other causes of $\mathrm{HF}$, especially coronary abnormalities. ${ }^{7}$

\section{Nuclear medicine}

The presence of cardiac inflammation may be demonstrated scintigraphy studies. ${ }^{16,38}$ In children with DCM, gallium-67 scintigraphy may be used to detect myocarditis, showing high sensitivity (87\%) and specificity (81\%) compared with EMB. ${ }^{43}$
However, this technique is not frequently used in children with suspected myocarditis. ${ }^{1,38}$

\section{Cardiac magnetic resonance}

Cardiovascular magnetic resonance is a tool widely accepted to investigate patients with suspected myocarditis and became the non-invasive diagnostic method of choice in the diagnosis of myocarditis. ${ }^{2,44,46}$ In pediatrics, in addition to contributing to diagnosis, cardiovascular magnetic resonance is able to contribute in prognosis, especially in cases of fulminant myocarditis. ${ }^{47}$ Martinez-Villar et al. ${ }^{45}$ showed the contribution of resonance in the diagnosis of myocarditis in adolescents with infarction-like presentation.

This technique allows for a detailed assessment of ventricular wall dimensions and thickness and of cardiac function; moreover, it provides data on myocardial tissue, showing the presence of edema, inflammation, and fibrosis. ${ }^{13}$ Edema is seen through enhancement of T2-weigthed image, early enhancement on T-1 weighted image is related to hyperemia. Conversely, late gadolinium enhancement translates the presence of fibrosis, which is typically not restricted to a vascular territory, being usually mesomural and subepicardial. ${ }^{2,13,32}$ Figures 2 and 3 illustrate these changes. Cardiac resonance is also able to identify segmental changes in ventricular walls, more commonly in inferolateral and basal walls. ${ }^{34}$

Friedrich et al. published in 2009 the Consensus on cardiovascular magnetic resonance for inflammatory cardiomyopathies, the Lake Louise criteria, which establish 
that the presence of edema, hyperemia, and necrosis/fibrosis are assessed through T2-weighted images, early enhancement, and late gadolinium enhancement; likewise, the presence of 2 or more of these criteria strongly suggest the presence of myocarditis, ${ }^{42}$ with a sensitivity of $67 \%$ and a specificity of $91 \% .{ }^{13}$ Diagnostic accuracy may be improved with myocardial assessment on T1-weighted images. ${ }^{47}$ Therefore, the use of T-1 mapping together with $\mathrm{T} 2$-weighted images and late gadolinium enhancement increases diagnostic accuracy to $96 \%{ }^{45,46}$

Others unspecific findings of myocarditis include: regional changes in ventricular wall motion, reduced myocardial function, and pericardial effusion. ${ }^{45}$

In addition to the diagnosis of myocarditis, magnetic resonance may provide information on disease progression and resolution. ${ }^{45}$

\section{EMB}

The histopathological demonstration defined by the Dallas criteria, proposed in $1986 .{ }^{14}$ Is still necessary for the definite diagnosis of myocarditis; thus, EMB is still the gold standard. 2,16,19 EMB shows the etiology of myocarditis, because infectious, inflammatory, toxic, infiltrative, and autoimmune changes occur at the cell level, and non-invasive methods are not able to demonstrate them. ${ }^{49}$ Although some factors limit its routine use, ${ }^{1,6,38}$ the indication of EMB is justified by the fact that specific inflammatory diseases of the myocardium have an unique treatment, impacting on prognosis. ${ }^{16,48,49-51}$ For example, the treatment of myocarditis mediated by immune processes differs from that of viral myocarditis, and it is difficult to diagnose them by non-invasive methods. ${ }^{1,49}$

The EMB procedure may rarely be related to some complications such as cardiac tamponade and atrioventricular block requiring temporary pacemaker.7,13,16 In pediatric patients, the overall risk of complications is relatively low, ranging from 1 to $6 \%,{ }^{1}$ and is directly related to patient's clinical condition, to the experience of the medical team in performing the procedure, and to the availability of cardiac surgery if needed..$^{38}$ Mortality rates
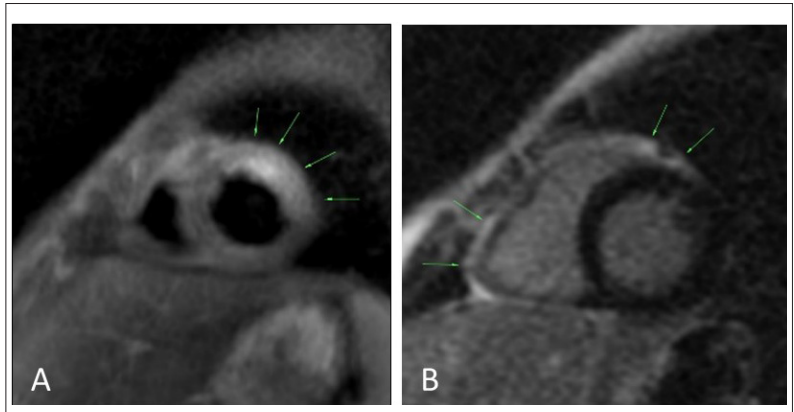

Figure 2 - Cardiac magnetic resonance showed myocardial edema at anterolateral mid-basal walls. (A) Effusion, thickening, and pericardial enhancement more evident near the right and left ventricular basal segments. (B) Aspect suggestive of recent or active myopericarditis. range from 0 to $0.4 \% .{ }^{1}$ Therefore, the risk/benefit of biopsy should be individually assessed.

The sensitivity of Dallas criteria for the diagnosis of myocarditis is around 55\%; however, the use of immunohistochemistry and polymerase chain reaction (PCR) for viral research increases the diagnostic sensitivity of $E M B,{ }^{13,38}$ since some studies showed the presence of virus in the absence of Dallas criteria. ${ }^{14,36,48}$ The collection EMB samples from both ventricles increases sensitivity for the diagnosis of myocarditis. ${ }^{43}$ The diagnosis may be improved with the combined use of EMB and magnetic resonance improve diagnosis, because magnetic resonance can guide the better site for the collection of EMB samples when focal lesions are present in the right or in the left ventricle. ${ }^{3,16,50}$

In 2007, a joint publication by the American Heart Association, the American College of Cardiology Foundation, and the European Society of Cardiology defined as class I recommendation for EMB two scenarios out of 14: unexplained new-onset HF of less than 2 weeks' duration associated with hemodynamic compromise; or unexplained new-onset HF of 2 weeks' to 3 months' duration associated with ventricular dilatation or arrhythmias or conduction disease. ${ }^{49}$ However, the European Society of Cardiology recently included the presence of pseudoinfarction as recommendation for EMB, after exclusion of coronary disease..$^{48}$ In the pediatric population, the presence of unexplained cardiomyopathy receives recommendation Ila for EMB. ${ }^{49}$

According to the Dallas criteria, acute myocarditis is defined by lymphocytic infiltrates in association with myocyte necrosis, whereas borderline myocarditis is characterized by inflammatory infiltrate without evidence of necrosis. ${ }^{51}$

\section{Treatment}

The therapeutic approach of myocarditis in childhood and adolescence is still controversial and mainly based on support treatment. ${ }^{1,12,20}$ However, specific treatment may be required, according to results from $\mathrm{EMB}$, clinical presentation, and disease prognosis. ${ }^{48}$

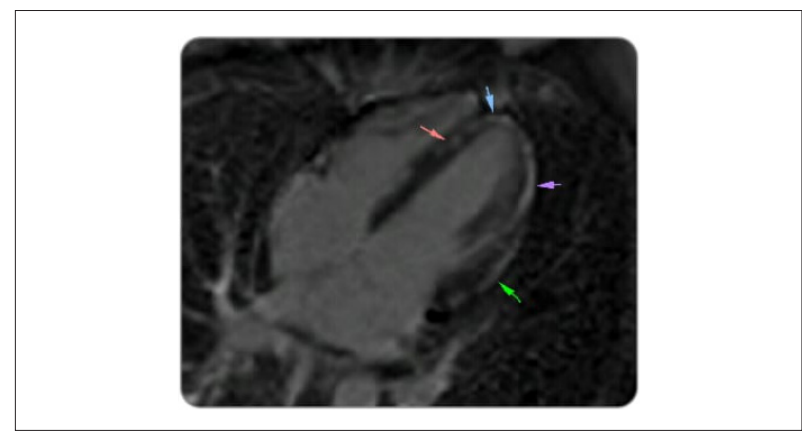

Figure 3 - Presence of atypical late enhancement for coronary disease with heterogeneous pattern, sparing the endocardium located in the anterior, anteroseptal, and inferior segments. Compatible with non-ischemic necrosis/fibrosis (myocarditis). 


\section{General measures}

These measures consist of treating HF and arrhythmias and of preventing sudden death by reducing myocardial oxygen consumption, treating anemia, and adhering to water and salt restriction with strict control of water balance. ${ }^{2,12}$

1. Physical activity limitation/rest: competitive activities should be suspended for 6 months, as long as ventricular function is normal and there is no evidence of clinically relevant arrhythmias. ${ }^{12,19}$

2. Treatment of HF: stabilizing children with hemodynamic compromise is crucial, admission to the pediatric intensive care unit for hemodynamic and ventilatory supports is required in cases presenting with HF.,11,12 Infusion of inotropics, use of afterload reducers, and use of diuretics help improve cardiac function.

The administration of diuretics should be made with caution, because rapid reduction in volemia may worsen hypotension and compromise tissue perfusion in a patient with an already reduced cardiac output. 2,11 The use of inotropics such as dobutamine, dopamine, noradrenaline, and adrenaline will be necessary in patients with hypotension, low cardiac output, and reduced cardiac function; their infusion may increase the risk for arrhythmias. The use of milrinone (phosphodiesterase inhibitor) is very useful in afterload reduction, improves cardiac function, and has a lower risk of predisposing to arrhythmias. , $^{21,19}$

The use de angiotensin-converting enzyme inhibitors (ACEI) and beta blockers in adult patients is associated with good prognosis. ${ }^{19}$ However, beta blockers should not be administered to children in the acute phase of myocarditis, when there is low cardiac output. After stabilization and transition to the use of an oral diuretic, the use of beta blockers such as carvedilol is recommended, as well as the use of ACEI, which is associated with reduced reverse remodeling and improved functional class. ${ }^{2}$ Digoxin should be used with extreme caution in patients in the acute phase of disease, since its administration may trigger arrhythmias and increase the production of inflammatory cytokines. ${ }^{11}$

3. Arrhythmias: in patients with myocarditis, arrhythmias are usually transient and occur during the acute phase of disease. However, changes in cardiac rhythm may be associated with greater mortality and should be managed conventionally. Despite that, some patients will require peacemaker implantation for total atrioventricular block, and those with sustained ventricular arrhythmia will require amiodarone and, in selected cases, implantable cardioverter defibrillator. ${ }^{11,49}$

4. Advance life support: mechanical circulatory support (MCS) is required in patients with refractory cardiogenic shock. MCS devices are able to replace heart's pumping function until myocardial function is recovered (rescue therapy) or until cardiac transplantation is possible (bridge therapy for transplantation)..$^{2,11,43}$

Pediatric patients with fulminant myocarditis benefit from ECMO support, because recovery of ventricular function may occur within some weeks. ${ }^{1,752}$

\section{Specific measures}

1. Intravenous immunoglobulin (IVIG): the use of immunoglobulin in pediatric patients with new-onset myocarditis or DCM is still controversial, since there are no available controlled studies showing its benefit. ${ }^{1,2,53-55}$ The use of immunoglobulin is based on its antiviral and immunomodulatory potential, which reduces the cytokine production and inflammation. ${ }^{1,7}$ Robinson et al. ${ }^{56}$ conducted a Cochrane review with several databases and concluded that IVIG may be useful in children with myocarditis associated encephalitis and that more controlled clinical trials are needed to demonstrate the benefit of IVIG in pediatric myocarditis. However, studies with series of pediatric cases show that the use of immunoglobulin improves ventricular function and survival in this population, suggesting that immunoglobulin has a beneficial role in pediatric myocarditis. ${ }^{1,7,49,54}$ In a meta-analysis study, Huang et al. ${ }^{53}$ demonstrated that patients with fulminant myocarditis showed better survival at follow-up and that the use of IVIG showed to be superior compared with conventional treatment in reducing in-hospital mortality rates. ${ }^{53}$ However, a systematic review with meta-analysis conducted by Yen et al. ${ }^{55}$ found that immunoglobulin did not change survival rates, and thus should not be routinely administered for acute pediatric myocarditis.

In the I Brazilian guideline on HF and cardiac transplantation in fetuses, children and adults with congenital heart disease, developed by the Brazilian Society of Cardiology (Sociedade Brasileira de Cardiologia, SBC), the use of IVIG is considered recommendation IIb, with level of evidence $\mathrm{B}$, for the following conditions: idiopathic DCM (optimized clinical treatment, more than 1 year with symptoms of HF) with presence of high titers of parvovirus B19 genome for in the myocardial tissue confirmed by EMB and molecular biology; myocarditis confirmed by EMB through immunohistochemistry and presence of viral genome in the myocardial tissue on molecular biology. ${ }^{43}$

2. Immunosuppression: the role of immunosuppression is still discussed in the treatment of myocarditis, with the purpose of suppressing inflammatory response and autoimmune activity in order to improve clinical status and ventricular function and reduce mortality. ${ }^{12,16,44,57}$ It may be considered adjuvant therapy when EMB is performed, and the presence of viral activity is ruled out through PCR study and documentation of inflammatory activity. ${ }^{11}$ The Tailored Immunosuppression in Inflammatory Cardiomyopathy (TIMIC) study confirms the efficacy of immunosuppressive therapy in this situation. ${ }^{57}$

In giant cell myocarditis confirmed by EMB, immunosuppression received class I recommendation I, level of evidence B. Conversely, in inflammatory cardiomyopathy confirmed by EMB through immunohistochemistry with optimized clinical treatment and more than 6 months of symptoms of HF, in the absence of viral genome by molecular biology, immunosuppression received class recommendation Ilb and level of evidence B. ${ }^{43}$ This therapy is also indicated in eosinophilic myocarditis. ${ }^{19}$ 
Camargo el al. ${ }^{58}$ showed that immunosuppressive therapy is beneficial in pediatric patients with inflammation on EMB, regardless of the presence of viral genome. However, their study did not use a control group of active patients with myocarditis treated conventionally. ${ }^{58}$

Therapy may be based on the association of prednisone and azathioprine or corticosteroids associated or not with cyclosporine. ${ }^{16,19,43}$

3. Antiviral therapy: the presence of viral genome in $E M B$ samples has guided antiviral therapy. Interferon- $\beta$ (IFN- $\beta$ ) and IVIG are the main therapeutic options, whose objective is to interrupt viral replication by eliminating them. It is necessary to confirm inflammatory activity associated with viral replication documented by EMB. ${ }^{16,49}$ Patients with DCM and persistent viral genome show improved cardiac function after using subcutaneous IFN- $\beta .^{7,49}$ The use of antiviral therapy is more effective in the earlier stages of myocarditis. ${ }^{19}$

Infections with cytomegalovirus, herpes simplex, and human immunodeficiency virus (HIV) should receive their predefined therapies. ${ }^{12}$

\section{Prognosis}

The prognosis of myocarditis is as variable as its clinical presentation. The patients may recover and subsequently evolve to DCM or death. ${ }^{44,59,60}$ Patients with normal cardiac function usually have good prognosis, unlike those with DCM. ${ }^{1,44}$ In general, nearly $50 \%$ of patients will improve after 2 to 4 weeks of disease onset, nearly $30 \%$ will develop DCM, and the remaining $20 \%$ may evolve to clinical worsening, requirement for cardiac transplantation, or death. ${ }^{17}$

Ejection fraction below $30 \%$ and shortening fraction bellow $15 \%$, LV dilatation, and moderate to severe mitral regurgitation are considered predictors of poor prognosis. ${ }^{1}$ In a study conducted by Kim et al., ${ }^{60}$ LV dilatation was the main factor associated with mortality in children with acute myocarditis. Transplantation-free survival in children with acute viral myocarditis ranges from 70 to $75 \% .{ }^{54}$ However, neonates and infants have significant mortality rates in up to $45 \%$ of the cases.

Patients with fulminant myocarditis are more likely to recover ventricular function after surviving the acute phase with cardiogenic shock. ${ }^{1,44}$

\section{New horizons}

New models of pathogen identification through metagenomic next-generation sequencing (mNGS) have contributed in the approach of myocarditis in large centers. In most studies with mNGS, it was observed that the identified viruses were not included in the routine viral panels. ${ }^{61,62}$ These studies also reinforce the need of population research fronts specific for epidemiology and etiology of myocardites. The pursuit of etiopathogenic diagnosis will contribute to characterize outcomes and prognosis in specific populations, thus enabling to include investigations with vaccines and appropriate antiviral therapeutics.

\section{Conclusions}

Myocarditis is characterized as a multifaceted disease able to promote different clinical pictures and outcomes. Numeral diagnostic and therapeutic technological advances have changed its natural history; however, epidemiological studies of clinical registries are essential to characterize the population in each country.

The Children and Adolescents Myocarditis and Cardiomyopathy Registry (ChARisMa), conducted by Universidade Federal Fluminense (UFF) in Niterói, state of Rio de Janeiro, Brazil, with partner institutions and the support from the SBC Department of Heart Failure (Departamento de Insuficiência Cardíaca da Sociedade Brasileira de Cardiologia, DEIC-SBC), aims to map and characterize pediatric and adolescent population with suspected or diagnosed myocarditis and cardiomyopathy. The obtained population data will contribute in guiding and developing protocols with educational, diagnostic, therapeutic, and follow-up measures.

Promoting research guidelines in diagnostic suspicion in childhood may contribute to better outcomes, not only in this population but also in the adult population with DCM of unknown etiology.

\section{Author Contributions}

Conception and design of the research and Critical revision of the manuscript for intellectual content: Torbey AFM, Souza ALAAG, Mesquita ET; Acquisition of data: Torbey AFM, Souza ALAAG, Bustamante ACD, Brandão CZ, Abdallah LR, Souza YPDM; Writing of the manuscript: Torbey AFM, Souza ALAAG, Bustamante ACD, Brandão CZ, Abdallah LR, Souza YPDM, Mesquita ET.

\section{Potential Conflict of Interest}

No potential conflict of interest relevant to this article was reported.

\section{Sources of Funding}

There were no external funding sources for this study.

\section{Study Association}

This article is part of the thesis of Doctoral submitted by Ana Flávia Malheiros Torbey e Aurea Lúcia Alves de Azevedo Grippa de Souza, from Programa de Pós-Graduação em Ciências Cardiovasculares da Universidade Federal Fluminense.

\section{Ethics approval and consent to participate}

This article does not contain any studies with human participants or animals performed by any of the authors. 


\section{References}

1. May LJ, Patton DJ, Fruitman DS. The evolving approach to paediatric myocarditis: a review of the current literature. Cardiol Young. 2011;21(3):241-51. doi: 10.1017/\$1047951110001964.

2. Singh RK, Yeh JC, Price JF. Diagnosis and treatment strategies for children with myocarditis. Prog Pediatr Cardiol. 2016;43:23-30. 10.1016/j. ppedcard.2016.08.022.

3. Towbin JA, Lowe AM, Colan SD, Sleeper LA, Orav EJ, Clunie S, et al. Incidence, causes, and outcomes of dilated cardiomyopathy in children. JAMA. 2006;296(15):1867-76. doi: 10.1001/jama.296.15.1867.

4. Durani Y, Egan M, Baffa J, Selbst SM, Nager AL. Pediatric myocarditis: presenting clinical characteristics. Am J Emerg Med. 2009;27(8):942-7. doi: 10.1016/j.ajem.2008.07.032.

5. Richardson P, McKenna W, Bristow M, Maisch B, Mautner B, O'Connell J, et al. Report of the 1995 World Health Organization/International Society and Federation of Cardiology Task Force on the Definition and Classification of cardiomyopathies. Circulation. 1996;93(5):841-2. doi: 10.1161/01. cir.93.5.841.

6. Rodriguez-Gonzalez M, Sanchez-Codez MI, Lubian-Gutierrez M, Castellano-Martinez A. Clinical presentation and early predictors for poor outcomes in pediatric myocarditis: a retrospective study. World J Clin Cases. 2019;7(5):548-61. doi: 10.12998/wjcc.v7.i5.548.

7. Blauwet LA, Cooper LT. Myocarditis. Prog Cardiovasc Dis. 2010;52(4):27488. doi: 10.1016/j.pcad.2009.11.006.

8. Arola A, Pikkarainen E, Sipilä JO, Pykäri J, Rautava P, Kytö V. Occurrence and features of childhood myocarditis: a nationwide study in Finland. J Am Heart Assoc. 2017;6(11):e005306. doi: 10.1161/JAHA.116.005306.

9. Simpson KE, Canter CE. Acute myocarditis in children. Expert Rev CardiovasC Ther. 2011;9(6):771-83. doi: 10.1586/erc.11.70.

10. Wu MH, Wu ET, Wang CC et al. Contemporary Postnatal Incidence of Acquiring Acute Myocarditis by Age 15 Years and the Outcomes From a Nationwide Birth Cohort. Pediatr Crit Care Med. 2017; (12):1153-1158

11. Merchant Q, Haque A, Hasan BS. Management of acute myocarditis in children. J Pak Med Assoc. 2013;63(6):803-11.

12. Howard A, Hasan A, Brownlee J, Mehmood N, Ali M, Mehta S, et al. Pediatric myocarditis protocol: an algorithm for early identification and management with retrospective analysis for validation. Pediatr Cardiol. 2020;41(2):31626. doi: 10.1007/s00246-019-02258-1.

13. Gannon MP, Schaub E, Grines CL, Saba SG. State of the art: evaluation and prognostication of myocarditis using cardiac mri. J Magn Reson Imaging. 2019;49(7):e122-e131. doi: 10.1002/jmri.26611.

14. Baughman KL. Diagnosis of myocarditis: death of Dallas criteria. Circulation. 2006;113(4):593-5. doi: 10.1161/CIRCULATIONAHA.105.589663.

15. Baughman KL. Diagnosis of myocarditis: death of Dallas criteria. Circulation. 2006;113(4):593-5. doi: 10.1161/CIRCULATIONAHA.105.589663.

16. ontera MW, Mesquita ET, Colafranceschi AS, Oliveira AC Jr, Rabischoffsky A, lanni BM, et al. I Brazilian guidelines on myocarditis and pericarditis. Arq Bras Cardiol. 2013;100(4 Suppl 1):1-36. doi: 10.5935/abc.2013S004.

17. Buggey J, ElAmm CA. Myocarditis and cardiomyopathy. Curr Opin Cardiol. 2018;33(3):341-46. doi: 10.1097/HCO.0000000000000514.

18. Kühl U, Schultheiss HP. Myocarditis in children. Heart Fail Clin. 2010;6(4):483-96. doi: 10.1016/j.hfc.2010.05.009.

19. Canter CE, Simpson KE. Diagnosis and treatment of myocarditis in children in the current era. Circulation. 2014;129(1):115-28. doi: 10.1161/ CIRCULATIONAHA.113.001372.

20. Levine MC, Klugman D, Teach SJ. Update on myocarditis in children. Curr Opin Pediatr. 2010;22(3):278-83. doi: 10.1097/MOP.0b013e32833924d2.
21. Alvarez MF, Bolívar-Mejía A, Rodriguez-Morales AJ, Ramirez-Vallejo E. Cardiovascular involvement and manifestations of systemic Chikungunya virus infection: a systematic review. F1000Res. 2017;6:390. doi: 10.12688/ f1000research.11078.2.

22. Grimaud M, Starck J, Levy M, Marais C, Chareyre J, Khraiche D, et al. Acute myocarditis and multisystem inflammatory emerging disease following SARS-CoV-2 infection in critically ill children. Ann Intensive Care. 2020;10(1):69. doi: 10.1186/s13613-020-00690-8.

23. Pirzada A, Mokhtar AT, Moeller AD. COVID-19 and myocarditis: what do we know so far? CJC Open. 2020;2(4):278-85. doi: 10.1016/j. cjco.2020.05.005.

24. Sanna G, Serrau G, Bassareo PP, Neroni P, Fanos V, Marcialis MA. Children's heart and COVID-19: Up-to-date evidence in the form of a systematic review. Eur J Pediatr. 2020 Jul;179(7):1079-1087

25. Dolhnikoff M, Ferranti JF, Monteiro RAA, Duarte-Neto AN, Gomes-Gouvêa MS, Degaspare NV, et al. SARS-CoV-2 in cardiac tissue of a child with COVID19-related multisystem inflammatory syndrome. Lancet Child Adolesc Health. 2020;4(10):790-94. doi: 10.1016/S2352-4642(20)30257-1.

26. Bajaj R, Sinclair HC, Patel K, Low B, Pericao A, Manisty C, et al. Delayed-onset myocarditis following COVID-19. Lancet Respir Med. 2021;9(4):e32-e34. doi: 10.1016/S2213-2600(21)00085-0.

27. Bracamonte-Baran W, Čiháková D. Cardiac Autoimmunity: Myocarditis. Adv Exp Med Biol. 2017;1003:187-221.doi: 10.1007/978-3-319-57613-8_10.

28. Simpson KE, Cunningham MW, Lee CK, Ward K, Tong A, Danon S, et al. Autoimmunity against the heart and cardiac myosin in children with myocarditis. J Card Fail. 2016;22(7):520-8. doi: 10.1016/j. cardfail.2016.02.009.

29. Bonaca MP, Olenchock BA, Salem JE, Wiviott SD, Ederhy S, Cohen A, et al. Myocarditis in the setting of cancer therapeutics: proposed case definitions for emerging clinical syndromes in cardio-oncology. Circulation. 2019;140(2):80-91. doi: 10.1161/CIRCULATIONAHA.118.034497.

30. Ryan TD, Nagarajan R, Godown J. Cardiovascular toxicities in pediatric cancer survivors. Cardiol Clin. 2019;37(4):533-44. doi: 10.1016/j. ccl.2019.07.002.

31. Neilan TG, Rothenberg ML, Amiri-Kordestani L, Sullivan RJ, Steingart RM, Gregory W, et al. Myocarditis associated with immune checkpoint inhibitors: an expert consensus on data gaps and a call to action. oncologist. 2018;23(8):874-78. doi: 10.1634/theoncologist.2018-0157.

32. Wisotzkey BL, Soriano BD, Albers EL, Ferguson M, Buddhe S. Diagnostic role of strain imaging in atypical myocarditis by echocardiography and cardiac MRI. Pediatr Radiol. 2018;48(6):835-42. doi: 10.1007/s00247017-4061-0.

33. Zhao L, FuZ. Rolesof hostimmunity inviral myocarditisand dilated cardiomyopathy. J Immunol Res. 2018;2018:5301548. doi: 10.1155/2018/5301548.

34. Etesami M, Gilkeson RC, Rajiah P. Utility of late gadolinium enhancement in pediatric cardiac MRI. Pediatr Radiol. 2016;46(8):1096-113. doi: 10.1007/ s00247-015-3526-2.

35. Bejiqi R, Retkoceri R, Maloku A, Mustafa A, Bejiqi H, Bejiqi R. The diagnostic and clinical approach to pediatric myocarditis: a review of the current literature. Open Access Maced J Med Sci. 2019;7(1):162-73. doi: 10.3889/ oamjms.2019.010.

36. Martin AB, Webber S, Fricker FJ, Jaffe R, Demmler G, Kearney D, et al Acute myocarditis. Rapid diagnosis by PCR in children. Circulation. 1994;90(1):330-9. doi: 10.1161/01.cir.90.1.330.

37. Sankar J, Khalil S, Sankar MJ, Kumar D, Dubey N. Short-term outcomes of acute fulminant myocarditis in children. Pediatr Cardiol. 2011;32(7):88590. doi: 10.1007/s00246-011-0007-8. 
38. Das BB. Role of endomyocardial biopsy for children presenting with acute systolic heart failure. Pediatr Cardiol. 2014;35(2):191-6. doi: 10.1007/ s00246-013-0807-0.

39. Testolina M, Schiavo A, Marcolongo R, Iliceto S. Endomyocardial biopsy should be performed in every patient with suspected myocarditis. G Ital Cardiol. 2015;16(10):533-8. doi: 10.1714/2028.22032.

40. Soongswang J, Durongpisitkul K, Nana A, Laohaprasittiporn D, Kangkagate C, Punlee K, et al. Cardiac troponin T: a marker in the diagnosis of acute myocarditis in children. Pediatr Cardiol. 2005;26(1):45-9. doi: 10.1007/ s00246-004-0677-6

41. Jeserich M, Konstantinides S, Pavlik G, Bode C, Geibel A. Non-invasive imaging in the diagnosis of acute viral myocarditis. Clin Res Cardiol. 2009;98(12):753-63. doi: 10.1007/s00392-009-0069-2.

42. Friedrich MG, Sechtem U, Schulz-Menger J, Holmvang G, Alakija P, Cooper LT, et al. Cardiovascular magnetic resonance in myocarditis: A JACC White Paper. J Am Coll Cardiol. 2009;53(17):1475-87. doi: 10.1016/j. jacc.2009.02.007.

43. Azeka E, Jatene MB, Jatene IB, Horowitz ESK, Branco KC, Souza Neto JD et al. I Diretriz Brasileira de Insuficiência Cardíaca e Transplante Cardíaco, no Feto, na Criança e em Adultos com Cardiopatia Congênita, da Sociedade Brasileira de Cardiologia. Arq Bras Cardiol 2014; 103(6Supl.2): 1-126

44. Tunuguntla H, Jeewa A, Denfield SW. Acute myocarditis and pericarditis in children. Pediatr Rev. 2019;40(1):14-25. doi: 10.1542/pir.2018-0044

45. Martinez-Villar M, Gran F, Sabaté-Rotés A, Tello-Montoliu A, Castellote A, Figueras-Coll $\mathrm{M}$, et al. Acute myocarditis with infarct-like presentation in a pediatric population: Role of cardiovascular magnetic resonance. Pediatr Cardiol. 2018;39(1):51-6. doi: 10.1007/s00246-017-1726-2.

46. Kotanidis CP, Bazmpani MA, Haidich AB, Karvounis C, Antoniades C, Karamitsos TD. Diagnostic accuracy of cardiovascular magnetic resonance in acute myocarditis: a systematic review and meta-analysis. JACC Cardiovasc Imaging. 2018;11(11):1583-90. doi: 10.1016/j.jcmg.2017.12.008.

47. Ferreira VM, Schulz-Menger J, Holmvang G, Kramer CM, Carbone I, Sechtem $\mathrm{U}$, et al. Cardiovascular magnetic resonance in nonischemic myocardial inflammation: Expert recommendations. J Am Coll Cardiol. 2018;72(24):3158-76. doi: 10.1016/j.jacc.2018.09.072.

48. Dominguez F, Kühl U, Pieske B, Garcia-Pavia P, Tschöpe C. Update on myocarditis and inflammatory cardiomyopathy: reemergence of endomyocardial biopsy. Rev Esp Cardiol. 2016;69(2):178-87. doi: 10.1016/j.rec.2015.10.015.

49. Cooper LT, Baughman KL, Feldman AM, Frustaci A, Jessup M, Kuh $U$, et al. The role of endomyocardial biopsy in the management of cardiovascular disease: a scientific statement from the American Heart Association, the American College of Cardiology, and the European Society of Cardiology. Circulation. 2007;116(19):2216-33. doi: 10.1161/ CIRCULATIONAHA.107.186093
50. Caforio ALP, Malipiero G, Marcolongo R, Iliceto S. Myocarditis: a clinical overview. Curr Cardiol Rep. 2017;19(7):63. doi: 10.1007/s11886-017-0870-x.

51. Kindermann I, Barth C, Mahfoud F, Ukena C, Lenski M, Yilmaz A, et al. Update on myocarditis. J Am Coll Cardiol. 2012;59(9):779-92. doi: 10.1016/j.jacc.2011.09.074

52. Xiong H, Xia B, Zhu J, Li B, Huang W. Clinical outcomes in pediatric patients hospitalized with fulminant myocarditis requiring extracorporeal membrane oxygenation: a meta-analysis. Pediatr Cardiol. 2017;38(2):209-214. doi: 10.1007/s00246-016-1517-1.

53. Huang X, Sun Y, Su G, Li Y, Shuai X. Intravenous immunoglobulin therapy for acute myocarditis in children and adults. Int Heart J. 2019;60(2):35965. doi: 10.1536/ihj.18-299.

54. Heidendael JF, Den Boer SL, Wildenbeest JG, Dalinghaus M, Straver B, Pajkrt D. Intravenous immunoglobulins in children with new onset dilated cardiomyopathy. Cardiol Young. 2018;28(1):46-54. doi: 10.1017/S1047951117001561.

55. Yen CY, Hung MC, Wong YC, Chang CY, Lai CC, Wu KG. Role of intravenous immunoglobulin therapy in the survival rate of pediatric patients with acute myocarditis: a systematic review and meta-analysis. Sci Rep. 2019;9(1):10459. doi: 10.1038/s41598-019-46888-0.

56. Robinson J, Hartling L, Vandermeer B, Klassen TP. Intravenous immunoglobulin for presumed viral myocarditis in children and adults. Cochrane Database Syst Rev. 2015;(5):CD004370. doi: 10.1002/14651858.CD004370.pub3. Update in: Cochrane Database Syst Rev. 2020;8:CD004370.

57. Frustaci A, Russo MA, Chimenti C. Randomized study on the efficacy of immunosuppressive therapy in patients with virus-negative inflammatory cardiomyopathy: the TIMIC study. Eur Heart J. 2009;30(16):1995-2002. doi: 10.1093/eurheartj/ehp249.

58. Camargo PR, Okay TS, Yamamoto L, Del Negro GM, Lopes AA. Myocarditis in children and detection of viruses in myocardial tissue: implications for immunosuppressive therapy. Int J Cardiol. 2011;148(2):204-8. doi: 10.1016/j.ijcard.2009.11.002

59. Di Filippo S. Improving outcomes of acute myocarditis in children. Expert Rev Cardiovasc Ther. 2016;14(1):117-25. doi: 10.1586/14779072.2016.1114884.

60. Kim G, Ban GH, Lee HD, Sung SC, Kim H, Choi KH. Leftventricular end-diastolic dimension as a predictive factor of outcomes in children with acute myocarditis. Cardiol Young. 2017;27(3):443-51. doi: 10.1017/S1047951116000706.

61. Kufner V, Plate A, Schmutz S, Braun DL, Günthard HF, Capaul R, et al. Two Years of Viral Metagenomics in a Tertiary Diagnostics Unit: Evaluation of the First 105 Cases. Genes. 2019;10(9):661. doi: 10.3390/genes10090661.

62. Takeuchi S, Kawada JI, Okuno Y, Horiba K, Suzuki T, Torii Y, et al. Identification of potential pathogenic viruses in patients with acute myocarditis using next-generation sequencing. J Med Virol. 2018;90(12):1814-21. doi: 10.1002/jmv.25263. 
